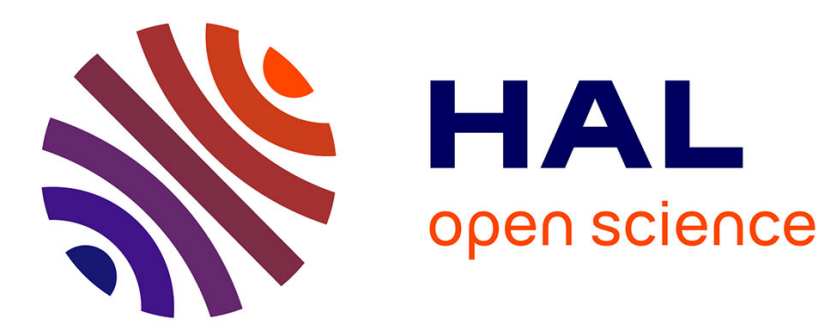

\title{
Sur l'observation comparative des raies telluriques et métalliques, comme moyen d'évaluer les pouvoirs absorbants de l'atmosphère
}

\author{
A. Cornu
}

\section{- To cite this version:}

A. Cornu. Sur l'observation comparative des raies telluriques et métalliques, comme moyen d'évaluer les pouvoirs absorbants de l'atmosphère. J. Phys. Theor. Appl., 1883, 2 (1), pp.58-63. 10.1051/jphystap:01883002005800 . jpa-00238173

\section{HAL Id: jpa-00238173 https://hal.science/jpa-00238173}

Submitted on 1 Jan 1883

HAL is a multi-disciplinary open access archive for the deposit and dissemination of scientific research documents, whether they are published or not. The documents may come from teaching and research institutions in France or abroad, or from public or private research centers.
L'archive ouverte pluridisciplinaire HAL, est destinée au dépôt et à la diffusion de documents scientifiques de niveau recherche, publiés ou non, émanant des établissements d'enseignement et de recherche français ou étrangers, des laboratoires publics ou privés. 


\section{SOR L'OBSERVATION COMPARATIVE DES RAIES TELLURIQUES ET MÉTAL- LIQUES, COMME MOYEN D'ÉVALUER LES POUVOIRS ABSORBANTS DE L'ATMOSPHËRE;}

PAR M. A. CORNU.

L'étude de l'absorption des radiations ultra-violettes par l'atmosphère m'a conduit naturellement à examiner la corrélation qui peut exister entre ce phénomène et l'absorption des radiations visibles.

Malheureusement, aux méthodes photographiques, dont les indications ont une sorte de valeur absolue, ne correspond aucune méthode photométrique pour la mesure absolue de l'intensité des radiations visibles : on ne peut procéder que par comparaison avec une source lumineuse parfaitement fixe comme éclat, suffisamment riche en radiations réfrangibles et dont l'obtention est difficile à réaliser.

Aussi m'a-t-il paru plus simple de chercher à utiliser un phénomène secondaire qui se produit en même temps que l'affaiblissement général des radiations, à savoir, l'existence dans le spectre solaire de bandes sombres (Brewster) désignées sous le nom de raies telluriques, dont l'intensité croît avec l'épaisseur atmosphérique traversée. Ces bandes, vues avec un spectroscope à faible dispersion, forment des teintes sombres sur diverses régions assez limitées du spectre : j'avais cherché d'abord à mesurer l'intensité comparative de deux régions voisines inégalement assombries par les bandes; mais les essais dans cette voie n'ont pas été satisfaisants : la différence de coloration des parties comparées, l'influence de la variation de la largeur de la fente du spectroscope rendent difficile toute détermination précise. Si, d'autre part, on augmente la dispersion pour pouvoir comparer des régions plus voisines comme coloration et pour atténuer l'influence de la fente, alors les bandes se résolvent en raies fines qui font disparaître l'uniformité des teintes à comparer.

La possibilité des mesures photométriques échappe donc encore de ce côté : j’ai finalement été réduit à rechercher simplement des repères destinés à distinguer dans l'absorption atmosphérique des degrés bien définis, d'après l'intensité des raies 
telluriques. Réduit à ces termes, le problème est beaucoup plus simple : il consiste à comparer des raies telluriques dont l'intensité est variable suivant l'épaisseur atmosphérique traversée par le faisceau observé, avec les raies fixes des éléments métalliques qui sillonnent également le fond continu du spectre solaire.

Cette méthode s’applique évidemment à toutes les bandes telluriques résolubles, quelle que soit la nature des substances qui les produisent : j'ai choisi de préférence l'un des groupes de bandes que les observations de notre confrère M. Janssen, d'Angström et d'autres physiciens ou météorologistes rapportent à l'action absorbante de la vapeur d'eau, le groupe voisin de la raie $D$ de Fraunhofer : ce groupe présente le double avantage d'offrir des variations considérables d'intensité sur une région extrèmement lumineuse, ce qui permet l'emploi d'un appareil assez dispersif pour résoudre les bandes en raies très fines.

La vapeur d'eau joue un rôle si considérable dans tous les phénomènes atmosphériques, et se présente en quantité si variable suivant les circonstances météorologiques, que l'étude de son influence s'impose avant celle de tous les autres éléments analogues.

Mon premier soin a été de dresser la Carte exacte des raies telluriques et métalliques de la région choisje : c'est le résultat de cette première étude que j'ai eu l'honneur de mettre sous les yeux de l'Académie, sous la forme d'une Carte spectrale, exprimée en longueurs d'onde, des raies comprises depuis $\lambda=\tilde{\jmath} 87,40$ jusqu'à $\lambda=60_{2}, 60$ (l'unité étant le millionième de millimètre); le nombre total de ces raies est d'environ 200 , dont 3 o raies métalliques $(\mathrm{Fe}$, $\mathrm{Ni}, \mathrm{Ti}, \mathrm{Mn}, \mathrm{Na}$ ) et г o raies telluriques.

L'échelle adoptée est quatre fois plus grande que celle des Planches du beau Mémoire d'Angström : une échelle plus petite permettait difficilement de représenter les détails avec assez de finesse ou de précision. Cette Carte a été obtenue par le relevé micrométrique (1) du quatrième spectre, fourni par réflexion sur un excellent réseau en verre argenté, dont les traits sont espacés

( ${ }^{1}$ ) La méthode employée est fondée sur le théorème suivant, qui ne me paraît pas avoir été signalé :

Si l'on observe l'un des spectres de diffraction dans le voisinage de la nor. male au plan d'un réseau fixe, les variations de la longueur d'onde des raies 
de $\mathrm{o}^{\mathrm{mm}}, \mathrm{oo}_{2} 935$ et que je dois à l'obligeance de M. Rutherfurd : les images (obtenues avec le collimateur et la lunette d'un petit cercle de MM. Brunner) sont assez parfaites pour qu'on ait avantage à remplacer l'oculaire de la lunette par un microscope composé grossissant vingt-cinq fois.

Les observations ont été faites à Courtenay (Loiret), pendant les mois d'octobre, novembre et décembre ı 8 79 , renouvelées pendant les années suivantes et, finalement, reprises et calculées à nouveau au mois d'octobre dernier.

J'ai pu vérifier, conformément à l'opinion des physiciens précités, que les raies telluriques de ce groupe présentent une intensité qui varie en raison composée de la quantité de vapeur d'eau contenue dans l'atmosphère et du chemin parcouru par le faisceau observé. Le phénomène a été particulièrement net pendant les grands froids de 1879 : je me bornerai à citer l'observation du 3 décembre faite par une température de $-\mathrm{II}^{\circ}:$ les raies telluriques voisines de $\mathrm{D}$, très intenses dans le spectre du Soleil, à son lever, à cause de la longueur du chemin parcouru par le faisceau, s'effacèrent en moins d'une heure, à mesure que le Soleil montait sur l'horizon, à tel point que les plus beaux groupes devinrent à

observées sont sensiblement proportionnelles aux variations de leurs distances angulaires relatives.

Le rapport de proportionnalité est égal au quotient de la distance constante a des traits du réseau par l'ordre du spectre observé.

Il en résulte que la connaissance de la valeur angulaire du tour de vis du micromètre permet de calculer la longueur d'onde de toutes les raies pointées dans le champ de la lunette, si l'on connaît celle de l'une d'elles en valeur absolue.

En effet, la distance angulaire $\delta$ d'une radiation diffractée $\lambda$, comptée, comme l'incidence $i$, d'un mème côté de la normale au plan du réscau, est donnée par la formule

$$
a(\sin i \pm \sin \delta)=n \lambda,
$$

\pm suivant qu'on observe par réflexion ou par transmission.

D'où l'on conclut, si l'incidence est constante,

$$
\frac{d \lambda}{d \delta}=\frac{a}{n} \cos \delta
$$

quotient qui se réduit à $\frac{a}{n}$ à moins de $\frac{\mathrm{I}}{1000}$ près en valeur relative, lorsque $\delta$ est compris entre $\pm 2^{\circ} 32^{\prime}$, c'est-à-dire dans un intervalle angulaire de plus de $5^{\circ}$.

Ce théorème, outre divers avantages purement optiques, réduit donc au minimum la difficulté du calcul d'interpolation, assez pénible dans la plupart des cas. 
peine visibles; le spectre ainsi dépouillé ne présentait plus que les raies métalliques.

Si l'on met à part ces cas exceptionnels d'une atmosphère desséchée par un froid intense, on peut dire que les raies telluriques principales sont toujours plus ou moins visibles pour un observateur exercé : on peut donc suivre la variation de leur assombrissement et pointer les heures ou mieux les hauteurs du Soleil qui correspondent à l'égalité de noirceur entre la raie choisie et les raies métalliques voisines. C'est un effet particulier, dépendant de la largeur de la raie et de l'énergie de l'absorption, que l'œil parvient à apprécier avec précision et dont il faut se contenter en l'absence de tout autre moyen de mesure. Tel est le principe de la méthode d'observation que je me permets de signaler à l'attention des physiciens et des météorologistes.

Voici la liste des principaux groupes de comparaison à recommander.

L'ordre des groupes correspond à une absorption croissante : les raies sont désignées par leur longueur d'onde ; le nombre entre parenthèses définit leur ordre relatif d'importance (de un à dix) : il est en chiffres arabes pour les raies métalliques fixes, en chiffres romains pour les raies telluriques variables.

Le croquis ci-joint, à petite échelle, qui reproduit l'aspect gé-

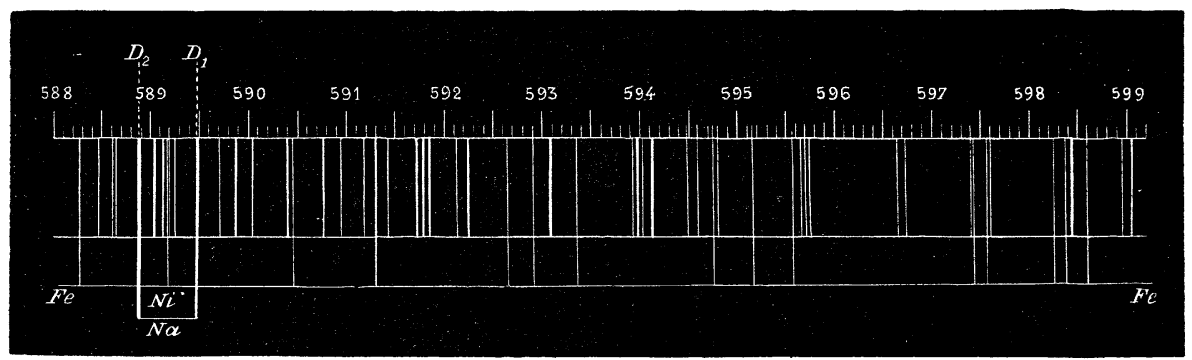

néral des groupes dont il va ètre question, facilitera la détermination de ces raies en attendant la publication définitive de la Carle ( ${ }^{1}$ ).

( $\left.{ }^{1}\right)$ Le dessin est actuellement à la gravure et sera publié, arec le Mémoirc détaillé, dans le Journal de l'École Polytechnique. 


$\begin{array}{ccc} & \text { Raies } & \\ \text { maies } & \text { détalli'jues } & \\ \text { telluriques. comparaison. } & \text { Remarques. }\end{array}$

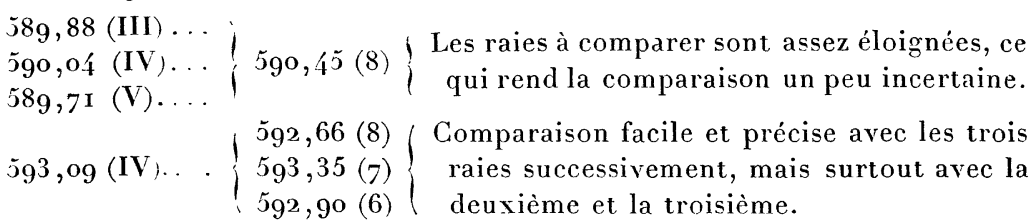

$\left.\begin{array}{l}59^{5,68}(\mathrm{VI}) . \ldots \\ 59^{5,72}(\mathrm{VI}) \ldots \\ 59^{5}, 76(\mathrm{VI}) \ldots .\end{array}\right) 595,57(7)\left\{\begin{array}{l}\text { Ce groupe de trois raies telluriques égales } \\ \text { et équidistantes (que je propose d'appeler, } \\ \text { pour abréger, le petit triplet) est très fa- } \\ \text { cile à retrouver et à observer. }\end{array}\right.$

59o,亿I (X) ... 59o,45 (8) Comparaison particulièrement précise.

$59 \mathrm{I}, 72$ (III) . . (Ce groupe de trois raies telluriques égales 5 yI, 78 (III) .... $59 \mathrm{I}, 30$ (4) et équidistantes (qu'on peut nommer le $59 \mathrm{I}, 84(\mathrm{III}) \ldots$. (grand triplet) est le plus reconnaissable 598,4 I (VIII) . . 598,3 $\quad$ (5) La raie tellurique est un peu estompée.

$589,88(\mathrm{IIJ}) . \cdots) \quad\left(589,48\right.$ est la raie $\mathrm{D}_{1}$; l'absorption est déjà э́go, $04(\mathrm{IV}) . \ldots\{589,48(2)\}$ très énergique; les raies telluriques com$589, \tau \mathrm{r}(\mathrm{V}) \ldots .$.$) \quad mencent à s'estomper sur les bords.$

Ces groupes de comparaison définissent des repères fixes dans la progression de l'absorption atmosphérique; chacun d'eux permet d'obtenir quelque chose de plus, à savoir : la mesure relative de la quantité totale de la substance absorbante qui produit la raie tellurique observée.

En effet, réduisons, pour simplifier, la forme des couches atmosphériques à des plans horizontaux; on aura, d'après la loi de Bouguer, pour la proportion $q$ de radiation transmise dans une direction faisant un angle $h$ avec l'horizon,

$$
q-a^{\frac{\varepsilon l}{\sin h}} \quad \text { ou } \log q=\frac{\varepsilon l}{\sin h} \log a
$$

$a$ étant le coefficient d'absorption de la radiation observée; $\varepsilon$ la quantité totale de substance absorbante; $l$ l'épaisseur du milieu absorbant dans la direction zénithale; si, en deux circonstances différentes, on observe, dans le spectre solaire, l'égalité d'intensité de la raie tellurique produite par cette absorption avec la 
même raie métallique aux deux hauteurs du Soleil $h, h^{\prime}$, on en conclura la relation

$$
\frac{\varepsilon}{\sin h}=\frac{\varepsilon^{\prime}}{\sin h^{\prime}}
$$

donc le rapport des quantités totales de matière absorbante est le rapport des sinus des hauteurs du Soleil pour lesquelles il y a égalité de la raie tellurique avec le même repère.

De là une méthode très simple pour évaluer relativement chaque jour et même à chaque heure la quantité totale de vapeur d'eau qui se trouve dans l'atmosphère : on aura évidemment autant de déterminations qu'on aura de couples d'observations d'un même repère.

Si l'on considère comme rigoureuse la loi exponentielle admise par les méléorologistes (voir Comptes rendus des séances de l'Académie des Sciences, t. XC, p. 9亿3) pour la loi de répartition de la vapeur d'eau avec l'altitude, on conclut aisément que $\varepsilon$ est proportionnel à la force élastique de la vapeur d'eau au lieu même d'observation, relation fort importante à bien des points de vue.

Il resterait à indiquer les conséquences qui dérivent de cette loi de répartition de la matière absorbante avec l'altitude, à parler de la comparaison des repères entre eux, de la variation séculaire des lignes métalliques fixes et de la possibilité de l'apprécier. Mais ces développements entraîneraient en dehors des limites d'unc simple Note : ils trouveront place dans le Mémoire détaillé.

L'exposé rapide qui précède suffit pour montrer les ressources que peut fournir aux physiciens et aux météorologistes la méthode d'observation dont je viens d'indiquer le principe : il n'est pas inutile d'ajouter qu'elle résout d'une manière, sinon parfaite, du moins fort simple, un problème dont on n'avait pas encore, à ma connaissance, donné de solution. 